\title{
LA DIFÍCIL DETERMINACIÓN DE LA EVOLUCIÓN DEL NÚMERO DE DÍAS DE TORMENTA EN ESPAÑA. EL CASO DE BARCELONA
}

\author{
Javier MARTÍN-VIDE y María del Carmen MORENO GARCÍA \\ Universidad de Barcelona
}

Recibido: 19/01/2013

Devuelto: $11 / 06 / 2013$

Aceptado: 16/06/2013

RESUMEN: En el artículo se recalca que la homogeneidad de una serie climática, que debe comprobarse con los tests de homogeneidad, es un requisito previo e indispensable para poderla analizar cronológicamente. La definición de día de tormenta da lugar a series frecuentemente inhomogéneas, por algunas dificultades en su aplicación. Al analizar los promedios anuales de días de tormenta de los períodos 1931-1960 y 1971-2000 de 62 observatorios españoles de primer orden de la red de AEMET se aprecia un muy acusado y casi general incremento entre el primero y el segundo que no puede atribuirse a un cambio climático. Una serie de calidad y homogénea del número anual de días de tormenta de casi un siglo (19172012) del observatorio Fabra (Barcelona) no ha mostrado tendencia alguna, a pesar del calentamiento reciente.

PALABRAS CLAVE: Barcelona, día de tormenta, homogeneidad, España.

THE DIFFICULT DETERMINATION OF THE EVOLUTION OF THE NUMBER OF DAYS OF STORM IN SPAIN. THE CASE OF BARCELONA

ABSTRACT: The paper highlights that the homogeneity of a climatic series is a previous and essential prerequisite so that it can be analyzed chronologically. The homogeneity has to be checked by means of the homogeneity tests. The definition of a thunderstorm day is not easy to be applied and gives series often inhomogeneous. The averages of the number of thunderstorm days of 62 Spanish stations, belonging to AEMET, for the periods 1931-1960 and 1971-2000 show big differences, with higher values in the last period, which can not be attributed to a climate change. A good quality, homogenous series of the annual number of thunderstorm days of the observatory Fabra (Barcelona) for the 96-year period 1917-2012 has not shown any statistically significant trend, despite the recent warming.

KEY WORDS: Barcelona, day of thunderstorm, homogeneity, Spain.

\section{INTRODUCCIÓN: EL PROBLEMA DE LA HOMOGENEIDAD DE LAS SERIES CLIMÁTICAS}

Uno de los asuntos metodológicos que ha adquirido más relevancia en los análisis temporales o cronológicos de las series climáticas es el de la homo- 
geneidad de las mismas, paso previo para el estudio de las posibles tendencias. Si una serie climática sistemática, larga y fiable, tras un control inicial de calidad de los datos que la componen, incluyendo el relleno de las eventuales lagunas y el rechazo de los outliers o valores imposibles, no es homogénea, debe rechazarse o someterse a un proceso de homogeneización. Sólo cuando se garantice la homogeneidad de la serie, se podrá proceder al análisis temporal de la misma. Este requisito previo y exigente en el análisis cronológico de series no siempre ha sido respetado, lo que ha dado lugar en el pasado a algunos resultados llamativos de presuntos cambios en el comportamiento temporal de ciertas variables climáticas en determinados lugares. Cambios que, evidentemente, no los podemos considerar como tales.

Una serie climática es homogénea cuando sus valores sólo reflejan el comportamiento natural (climático) de la correspondiente variable, sin interferencias o perturbación por hechos artificiales, es decir sus variaciones son fruto exclusivo de variaciones en el clima (MARTín VIDE, 2003; AGUILAR et al. 2003). Las principales causas de inhomogeneidad en una serie climática son: (1) cambios en el emplazamiento de la estación meteorológica; (2) modificación del entorno del lugar de observación; (3) cambios de los instrumentos de medida; (4) errores instrumentales y el deterioro progresivo de los aparatos; (5) modificación de los horarios de observación; (6) variaciones en los sistemas de observación y en el procedimiento de cálculo; y (7) la urbanización progresiva del área donde se ubica la estación meteorológica. Así, la mudanza del emplazamiento de la estación meteorológica, que no es rara, puede afectar de inmediato a sus registros. Por ejemplo, en el caso de la presión atmosférica, si el nuevo lugar no está a la misma altitud, se aprecia un escalón brusco en los registros. La modificación del entorno del lugar, con la construcción de edificios, muros, etc. o el crecimiento de vegetación, puede perturbar, por ejemplo, los registros de temperatura, de viento o de insolación, por el obstáculo directo que suponen para los rayos solares o el aire en movimiento. El cambio de un aparato meteorológico por otro, sea incluso del mismo modelo, puede introducir algunas variaciones, por los errores y diferencias inherentes a cada unidad. La sustitución de estaciones manuales por automáticas introduce con frecuencia saltos en las mediciones. Los errores instrumentales son relativamente frecuentes, obligando a una calibración sistemática. El deterioro progresivo de los aparatos o de sus escalas puede añadir gradualmente un error a los registros, marcando una cierta tendencia, al alza o a la baja. La modificación de los horarios de observación de las variables y de los fenómenos meteorológicos puede dar lugar a valores y frecuencias diferentes. Sería el caso, por ejemplo, de la observación de ciertos meteoros, como la niebla, que, si es de irradiación, muestra una mayor presencia a primeras horas de la mañana, o incluso de la nubosidad en general. Los 
cambios en los sistemas y protocolos de observación, así como en el procedimiento de cálculo de los valores estadísticos de los registros, también puede introducir sesgos respecto a los registros previos. Como ejemplo, el cálculo de la temperatura media como semisuma de las temperaturas máxima y mínima difiere algo de la hallada a partir de las temperaturas de las 0, 6, 12 y 18 h., o de todas las horarias. Finalmente, la urbanización progresiva, englobando a veces en su seno a las estaciones meteorológicas más antiguas, se visualiza por un aumento térmico gradual, causado por la intensificación de las islas de calor.

Por lo dicho anteriormente, las inhomogeneidades se visualizan en las gráficas temporales por un salto brusco, o break point (punto de ruptura) o por una cierta tendencia. El primer tipo de inhomogeneidad resulta fácilmente identificable como tal, pudiéndose, además, señalar con precisión el momento en que aparece el comportamiento diferente o anómalo, que coincide exactamente con el punto de ruptura de la serie. Las inhomogeneidades que producen cambios graduales o tendencias son mucho más difíciles de identificar como tales, pudiéndose confundir con auténticas tendencias en el comportamiento del clima. Estas inhomogeneidades son, así, mucho más sutiles y difíciles de discriminar de los verdaderos cambios y variaciones climáticas. En todo caso, hay que saber que un alto porcentaje de las series climáticas están afectadas por inhomogeneidades e irregularidades (AUER et AL, 2005), que debieran ser corregidas antes de abordar su análisis temporal.

Con vistas a determinar del modo más objetivo y preciso posible si una serie es homogénea o no, es decir, en este último caso si presenta inhomogeneidades, se utilizan los llamados tests de homogeneidad y se consulta la metadata o metadatos. Esto último refiere a las informaciones sobre la toma de los datos, los tipos de instrumental y sus modelos, los horarios, los eventuales cambios en el entorno y en el propio personal de observación, etc. Se trata de una información muy valiosa, pero a menudo difícil de encontrar. A veces, ni tan siquiera existió. Algunos climatólogos han actuado como auténticos detectives en la búsqueda de los metadatos, tarea más propia en un principio de un especialista en información y documentación. La metadata sirve en algunos casos para confirmar y explicar el motivo de una cierta inhomogeneidad.

Los tests de homogeneidad analizan numéricamente los datos que componen la serie objeto de estudio y deciden, mediante ciertos parámetros estadísticos, si es homogénea o no. Se suelen dividir en dos grandes grupos: de homogeneidad absoluta y de homogeneidad relativa. Los tests del primer grupo determinan si la serie analizada es homogénea o no sin recurrir a una o más series patrón o de referencia homogéneas. Realizan ciertas operaciones ma- 
temáticas exclusivamente con los datos de la serie analizada decidiendo así sobre su homogeneidad. En el caso de los tests de homogeneidad relativa el peso del procedimiento recae en la comparación de la evolución de la serie objeto de estudio con una o varias buenas, es decir, presuntamente homogéneas de referencia, próximas. Se supone en este caso que la evolución del clima de la región en que se ubican todas las series es la misma, de manera que, si la analizada no mantiene "correspondencia" temporal con las de referencia, es inhomogénea. Los tests de homogeneidad relativa son más robustos que los de homogeneidad absoluta, pero, como ventaja, estos últimos pueden ser aplicados a cualquier serie, sin el requisito de otras series. La Organización Meteorológica Mundial vino recomendando durante mucho tiempo el test de Thom, o de las rachas o de las alternancias, que es de homogeneidad absoluta. Conviene añadir que el análisis de la homogeneidad de una serie no se suele realizar a una resolución más fina que la mensual. El lector puede ampliar estas consideraciones en PETERSON et al. (1998), WiJNGAARD et al. (2003), AGUILAR et al. (2003), DUCRÉ-ROBITAILLE et al. (2003), REEVES et al. (2007) y COSTA y SOARES (2009), entre otros.

Entre los tests de homogeneidad más conocidos están los de Thom (THOM, 1966), Von Neumann (VON NEUMANN, 1941), Buishand (BUISHAND, 1982), Kruskal-Wallis (KRUSKAL y WALLIS, 1952), Mann-Kendall (MANN,1945; Kendall, 1975), Mash (SzentimRey, 2003), Pettitt (PetTitT, 1979), de regresión (SOLOW, 1987; EASTERLING y PETERSON, 1995; etc), Craddock (CRADDOCK, 1979), Standard Normal Homogeneity Test o SNHT (AlEXANDERSSON 1986; AleXANDERSSON y MOBERG 1997), WilcoxonMann-Whitney (MANN y WHITNEY, 1947; WILCOXON, 1945), y otros (HIRSCH y SLACK, 1984; VINCENT, 1998; MENNE y WiLLIAMS, 2005, etc).

Para homogeneizar las series que presentan puntos de ruptura se calculan las medias de las subseries antes del punto de ruptura y después del mismo y se aplica como factor de corrección su diferencia (por ejemplo, en el caso de la temperatura y de la presión atmosférica) o su cociente (por ejemplo, en la precipitación) a la subserie problemática. Si la inhomogeneidad se traduce en tendencias, se utiliza como factor de corrección la pendiente de la serie de diferencias o de cocientes entre la serie analizada y la de referencia (COSTA y SOARES, 2009).

Sin embargo, note el lector para finalizar, que cabe la posibilidad de que los tests de homogeneidad califiquen de inhomogéneas series homogéneas con tendencias naturales. Eso llevaría a homogeneizar o corregir series buenas que no lo necesitan, con el resultado de una suavización de las tendencia naturales. Por ello, como recomendación, conviene conservar siempre la serie de datos originales, aun no siendo homogénea, por si en el futuro hubiera 
nuevos procedimientos o técnicas de análisis, reconstrucción u homogeneización de series.

\section{CONSIDERACIONES SOBRE LA VARIABLE NÚMERO DE DÍAS DE TORMENTA}

A diferencia del número de días de otros fenómenos meteorológicos, como la lluvia, la nieve, el granizo, etc, el número de días de tormenta cuenta con un problema observacional de partida que puede afectar significativamente a su valor. En Meteorología, tormenta se define estrictamente como una o más descargas bruscas de electricidad atmosféricas acompañadas por un destello luminoso (relámpago) y un ruido seco o retumbante (trueno). Las descargas en sí son los rayos. Pero mientras la lluvia o la nieve han de caer en el mismo lugar de observación para que sean anotados en los diarios meteorológicos, hecho además fehaciente por la cantidad acumulada en el pluviómetro, las descargas o rayos no han de caer, naturalmente, en ese preciso lugar para que el citado electrometeoro sea registrado, y para que, en consecuencia, se contabilice un día de tormenta. En qué elemento objetivo, pues, hay que basarse para anotar tormenta en un diario meteorológico. Naturalmente, en una de sus dos manifestaciones (relámpago o trueno) o en las dos al tiempo. La observación del relámpago, en tormentas con muy poca actividad eléctrica puede pasar inadvertida para un observador, por su brevedad, e, incluso, no percibirse en días luminosos. Esto es relativamente frecuente en las horas centrales de jornadas de verano. $\mathrm{Y}$, por otra parte, desde observatorios con buena vista, sean de montaña o junto al mar, los relámpagos de tormentas nocturnas muy lejanas, a varias decenas de kilómetros, pueden percibirse aunque sea muy débilmente. El trueno, por el contrario, es un elemento de la tormenta cuya percepción es más segura, se oye de día y de noche, y "próxima”, en el sentido de que los truenos de tormentas muy lejanas, aun viéndose sus relámpagos, no son audibles. Este último hecho acota la consideración de si hubo tormenta o no en un lugar y sus proximidades mejor que la visión del relámpago. Aun así, de noche, sin ruido ambiental, pueden llegar a oírse truenos de tormentas hasta unos $20 \mathrm{~km}$ de distancia o más del lugar de audición (los autores de este trabajo han contabilizado en alguna ocasión hasta 60 segundos entre el relámpago y el trueno con alguna tormenta nocturna lejana, lo que equivale a una distancia de unos $20 \mathrm{~km}$ ). Por otra parte, en espacios urbanos, aeroportuarios, autopistas con tráfico denso o en general lugares ruidosos los truenos relativamente próximos pueden quedar enmascarados por el ruido ambiental. En resumen, ninguna de las dos manifestaciones de la tormenta permite una observación totalmente objetiva, homogénea y acotada a un entorno razonable del lugar de observación. En Meteorología se consigna tormenta cuando se ha percibido al menos un trueno. Sin 
embargo, en algunas estaciones meteorológicas se ha usado un criterio bastante más restrictivo, como el que se haya producido al menos un relámpago en el que su diferencia con el correspondiente trueno no supere los 10 segundos. Esto implica que una parte de la descarga eléctrica haya pasado a una distancia de no más de 3,4 km del observador $(340 \mathrm{~m} / \mathrm{s}$-velocidad del sonido- por $10 \mathrm{~s}$ ). Se trata en este último caso de tormentas realmente próximas. El criterio oficial tiene la ventaja de que el observador cubre una amplia área de su entorno, aunque la desventaja de que, por lo dicho, los registros de un lugar y otro serán difícilmente comparables. Imagine el lector, en este sentido, truenos muy lejanos en una tormenta nocturna. No es raro que el fenómeno pase inadvertido para muchos observadores únicos durmiendo. Un criterio más estricto, como el de los 10 segundos u otro parecido, no contabilizaría un estimable número de tormentas, pero permitiría la comparación de los registros de muchos observatorios.

Las redes de descargas eléctricas, como la de AEMET en el caso de España, pueden hoy precisar de forma objetiva el número de días de tormenta de una población analizando la proximidad de la localización de los rayos a ese lugar. Esas observaciones no disponen aún de un período suficientemente largo como para atribuir, de entrada, significación climática a sus resultados. A pesar de ello, los mapas ya construidos con los datos disponibles reflejan ya bien la distribución espacial de las tormentas, con el cuadrante nordeste de la Península Ibérica como el área con mayor frecuencia de tormentas, en particular el Pirineo oscense, en que se llega a rebasar los 40 días en promedio anual, seguido por el catalán, así como el tramo final turolense de la cordillera Ibérica (sierra de Gúdar, en particular). Las provincias con menor número de días de tormenta son las andaluzas, si se exceptúan las islas Canarias, que tienen tan solo 2 o menos días de tormenta en promedio anual.

En el excelente trabajo de Jorge González Márquez Climatología de tormentas en España, publicado por entregas en el portal Meteored (www.meteored.com) y completo en Divulgameteo (www.divulga meteo.com), a partir del período de diez años de octubre de 1997 a septiembre de 2006, se realiza un análisis a nivel provincial y se construyen dos mapas, uno del número medio anual de días de tormenta (FIGURA 1), suficientemente explícito y al que hay que atribuir significación climática por su coherencia geográfica, y otro del número medio anual de días de tormenta fuerte. El citado autor consideró día de tormenta en un determinado punto como aquel en el que se registra al menos una descarga eléctrica en un radio de $10 \mathrm{~km}$ alrededor del observatorio, lo que justifica principalmente por el hecho de que los truenos se suelen oír bien hasta esa distancia (máximo de poco más de treinta segundos entre el relámpago y el trueno). 
Figura 1. Mapa del número medio anual de días de tormenta

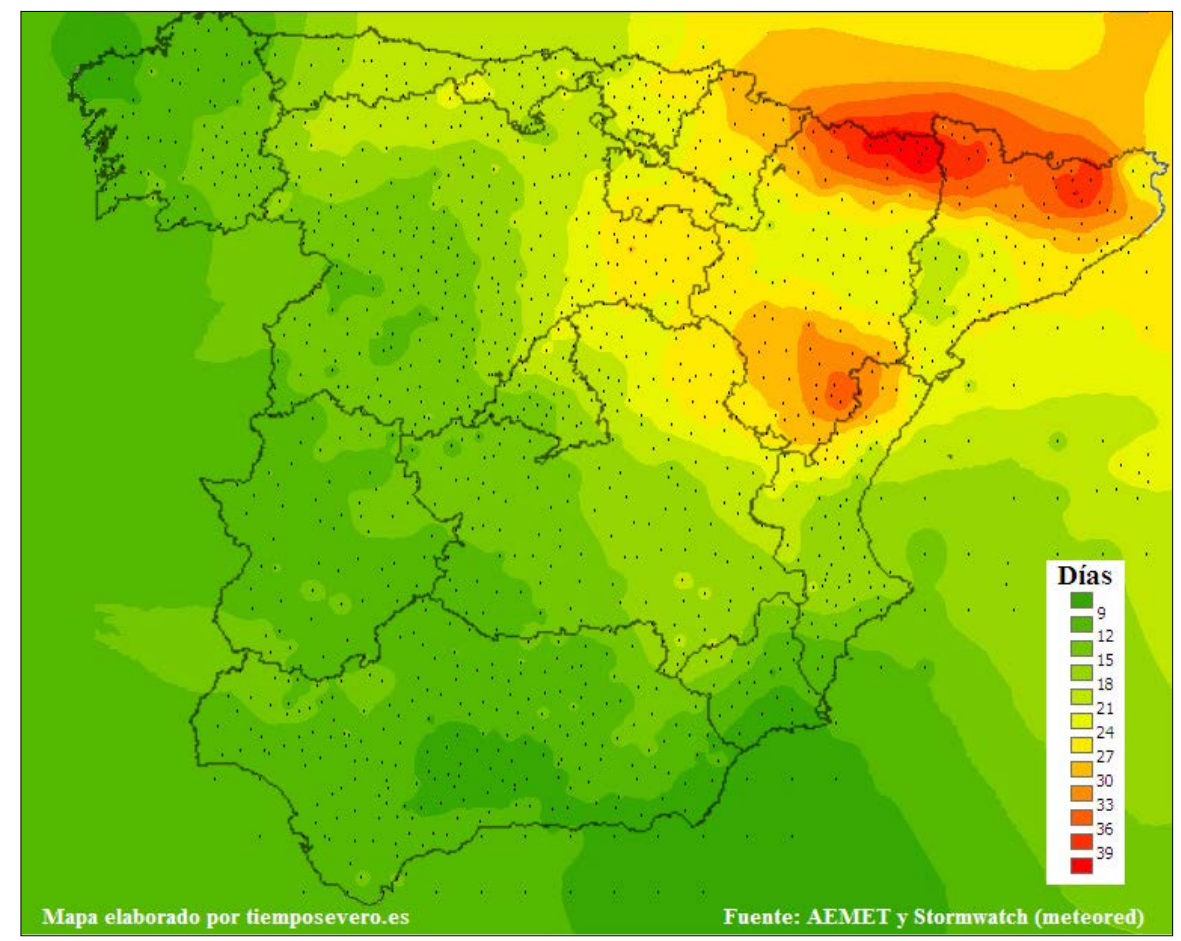

FuENTE: Extraído del trabajo de Jorge GonZÁLEz MÁRQUEZ, Climatología de tormentas en España.

\section{LA EVOLUCIÓN DEL NÚMERO ANUAL DE DÍAS DE TORMENTA EN ESPAÑA}

Dadas las consideraciones expuestas anteriormente, no parece fácil la tarea de analizar temporalmente las series de número de días de tormenta. Para minimizar la subjetividad, las deficiencias y los cambios de observadores y criterios en el registro de las tormentas, se han tomado los observatorios de primer orden de la Agencia Estatal de Meteorología (AEMET), y sus precedentes Instituto Nacional de Meteorología y Servicio Meteorológico Nacional, de dos treintenios, el internacional 1931-1960 y el 1971-2000, cuyos valores medios anuales (y mensuales) figuran en INM (1982) y en www.aemet.es, respectivamente. Se han comparado los valores de los dos treintenios en un total de 62 observatorios. En 16 de ellos (un 26,2\%) el emplazamiento del observatorio del lugar considerado no es el mismo entre un treintenio y el otro, aunque son próximos. En el CUADRO 1 se recogen los citados valores. 
Cuadro 1. Número medio anual de día de tormenta en 62 observatorios de España, en los períodos 1931-1960 y 1971-2000, y variaciones absoluta y porcentual.

\begin{tabular}{|c|c|c|c|c|}
\hline & $\begin{array}{c}A \\
1931-1960\end{array}$ & $\begin{array}{c}B \\
1971-2000\end{array}$ & $\Delta(B-A)$ & $\Delta(B-A) \%$ \\
\hline A Coruña & 9 & 16 & 7 & 77,8 \\
\hline Albacete Los Llanos & 13 & 20 & 7 & 53,8 \\
\hline Alcantarilla & 9 & 11 & 2 & 22,2 \\
\hline Alicante Ciudad Jardín & 11 & 14 & 3 & 27,3 \\
\hline Almería Ciudad Jardín & 8 & 8 & 0 & 0,0 \\
\hline Arrecife & 1 & 2 & 1 & 100,0 \\
\hline Ávila & 13 & 13 & 0 & 0,0 \\
\hline Badajoz & 10 & 13 & 3 & 30,0 \\
\hline Barcelona & 13 & 22 & 9 & 69,2 \\
\hline Bilbao Sondica & 13 & 24 & 11 & 84,6 \\
\hline Burgos Villafría & 15 & 18 & 3 & 20,0 \\
\hline Cáceres & 12 & 12 & 0 & 0,0 \\
\hline Castellón de la Plana & 14 & 21 & 7 & 50,0 \\
\hline Ceuta & 8 & 9 & 1 & 12,5 \\
\hline Ciudad Real & 12 & 17 & 5 & 41,7 \\
\hline Córdoba & 9 & 12 & 3 & 33,3 \\
\hline Cuenca & 14 & 19 & 5 & 35,7 \\
\hline Gijón & 14 & 17 & 3 & 21,4 \\
\hline Girona & 24 & 24 & 0 & 0,0 \\
\hline Granada Armilla & 10 & 11 & 1 & 10,0 \\
\hline Huelva & 7 & 9 & 2 & 28,6 \\
\hline Huesca Monflorite & 21 & 23 & 2 & 9,5 \\
\hline Ibiza Es Codolà & 11 & 15 & 4 & 36,4 \\
\hline Izaña & 3 & 3 & 0 & 0,0 \\
\hline Las Palma Gando & 3 & 2 & -1 & $-33,3$ \\
\hline León & 16 & 16 & 0 & 0,0 \\
\hline Lleida & 14 & 18 & 4 & 28,6 \\
\hline Logroño Agoncillo & 25 & 19 & -6 & $-24,0$ \\
\hline Lugo Instituto & 10 & 15 & 5 & 50,0 \\
\hline Madrid Barajas & 14 & 17 & 3 & 21,4 \\
\hline Madrid Retiro & 11 & 16 & 5 & 45,5 \\
\hline Mahón & 17 & 26 & 9 & 52,9 \\
\hline Málaga Rompedizo & 10 & 12 & 2 & 20,0 \\
\hline Melilla & 2 & 14 & 12 & 600,0 \\
\hline Molina de Aragón & 26 & 25 & -1 & $-3,8$ \\
\hline Navacerrada & 9 & 22 & 13 & 144,4 \\
\hline Ourense & 5 & 14 & 9 & 180,0 \\
\hline Oviedo & 2 & 20 & 18 & 900,0 \\
\hline Palma de Mallorca & 11 & 17 & 6 & 54,5 \\
\hline Pamplona & 14 & 23 & 9 & 64,3 \\
\hline
\end{tabular}




\begin{tabular}{|c|c|c|c|c|}
\hline Ponferrada & 10 & 13 & 3 & 30,0 \\
\hline Salamanca Matacán & 14 & 16 & 2 & 14,3 \\
\hline San Javier & 10 & 13 & 3 & 30,0 \\
\hline San Sebastián Igueldo & 21 & 31 & 10 & 47,6 \\
\hline Sta Cruz de Ten. Los Ro. & 2 & 3 & 1 & 50,0 \\
\hline Santa Cruz de Tenerife & 2 & 2 & 0 & 0,0 \\
\hline Santander & 18 & 14 & -4 & $-22,2$ \\
\hline Santiago Universidad & 7 & 13 & 6 & 85,7 \\
\hline Segovia & 11 & 14 & 3 & 27,3 \\
\hline Sevilla Tablada & 7 & 9 & 2 & 28,6 \\
\hline Soria & 18 & 23 & 5 & 27,8 \\
\hline Tarifa & 11 & 15 & 4 & 36,4 \\
\hline Toledo & 15 & 15 & 0 & 0,0 \\
\hline Tortosa & 33 & 25 & -8 & $-24,2$ \\
\hline Valencia & 14 & 18 & 4 & 28,6 \\
\hline Valladolid Villanubla & 12 & 16 & 4 & 33,3 \\
\hline Valladolid & 17 & 17 & 0 & 0,0 \\
\hline Vigo & 14 & 16 & 2 & 14,3 \\
\hline Vitoria Instituto & 12 & 21 & 9 & 75,0 \\
\hline Zamora & 9 & 15 & 6 & 66,7 \\
\hline Zaragoza & 10 & 21 & 11 & 110,0 \\
\hline
\end{tabular}

FUENTE: Elaboración propia, a partir de INM (1982) y de www.aemet.es.

De la simple inspección del cuadro se aprecia una variación muy sustancial al alza, en la mayoría de los observatorios, de los valores del primero al segundo período analizado, imposible de aceptar como cambio natural. 47 observatorios (el 77,0\%) presentan un valor más elevado en el último treintenio que en el primero, 9 no muestran variación y solo 5 tienen un valor inferior en el segundo período. Pero más que el elevado número de observatorios con valores al alza, que podría ser posible de forma natural, es la variación absoluta (y porcentual en los casos con valores moderados o altos del número de días de tormenta) lo más llamativo, con cambios muy grandes para los que hay que descartar totalmente una causa natural. Zaragoza, Navacerrada y Ourense duplican con creces su valor al pasar del primer período al segundo, y Melilla y Oviedo presentan variaciones extraordinarias que solo cabe achacar a graves deficiencias en la observación, errores en los registros o erratas en el treintenio 1931-1960. Otras variaciones son también muy sustanciales, pero nada justifica concluir en que las tormentas se hayan hecho más frecuentes en España a lo largo del siglo XX, o, con más precisión, al pasar del primer período analizado al segundo. Muy probablemente los criterios de observación de las tormentas en el treintenio 1931-1960 fueron más laxos que en el 1971-2000. Los valores del período 1971-2000 aquí presentados se asemejan a los calculados por González Márquez en su estudio. 
Otras observaciones puntuales permiten también reafirmar la relativa bondad de los valores medios anuales de días de tormenta del período 1971-2000 y la escasa fiabilidad, por defecto, de los correspondientes al período 19311960, salvo excepciones.

Ante tales hechos, cómo estudiar la evolución temporal de número de días de tormenta en España a lo largo de un período secular. El procedimiento más seguro es analizar solo las series de observatorios con registros largos y de calidad, y, especialmente, cuyas observaciones y criterios meteorológicos se hayan mantenido prácticamente inalterados a lo largo del tiempo. Un ejemplo de ello es el observatorio Fabra de Barcelona.

\section{EL CASO DEL OBSERVATORIO FABRA DE BARCELONA}

El observatorio Fabra, perteneciente a la Real Academia de Ciencias y Artes de Barcelona, se halla situado en la vertiente sureste de la sierra de Collserola $\left(41,42^{\circ} \mathrm{N} ; 2,12^{\circ} \mathrm{E}\right)$, a $413 \mathrm{~m}$ de altitud, cerca de la cima del Tibidabo, en el municipio de Barcelona. Se trata, pues, de un observatorio que no es propiamente urbano, por su localización en el parque natural de la citada sierra. El observatorio está próximo a cumplir su centenario, dado que comenzó a funcionar en 1914. Su sección meteorológica cuenta con registros sistemáticos de alta calidad y sin lagunas de prácticamente todas las variables meteorológicas, con historiales y metadata completos. Su privilegiada ubicación, con unas excelentes vistas, sobre todo hacia la vertiente marítima, cubriendo desde el rumbo nordeste al sudoeste, y la dedicación, preparación y experiencia del personal a su cargo, así como el prestigio científico de la institución a la que pertenece, explican la calidad y continuidad de sus series climáticas.

En el ANEXO 1 se recoge el número anual de días de tormenta del período 1917 a 2012 (se han excluido los valores de los tres primeros años de funcionamiento, cuyos datos son incompletos o anómalos). En conjunto se dispone de una serie de 96 años.

El promedio anual de días de tormenta es 16,7, con un máximo de 39 días, en 1971, y un mínimo de 7 días, en 1987. La mediana se acerca mucho a la media, 16,5 días. La desviación típica es 5,2 días. Un 25\% de los años contabilizó 13 o menos días de tormenta (primer cuartil) y otro porcentaje igual registró más de 19,2 días (tercer cuartil). Los meses que aportan más días de tormenta al total anual son septiembre y agosto, con 3,1 y 2,9 días, en promedio, respectivamente, fruto de la convección característica del verano y de los procesos ciclogenéticos otoñales, mientras que de noviembre a marzo, inclusive, el promedio mensual es inferior a 1 día. El promedio anual de días 
de tormenta hallado para el observatorio Fabra está comprendido entre los valores de los dos períodos analizados en el apartado anterior para Barcelona. En la FIGURA 2 se representa la serie temporal original y una suavizada.

Figura 2. Serie temporal del número anual de días de tormenta del observatorio Fabra (Barcelona)(1917-2012), con una suavización exponencial

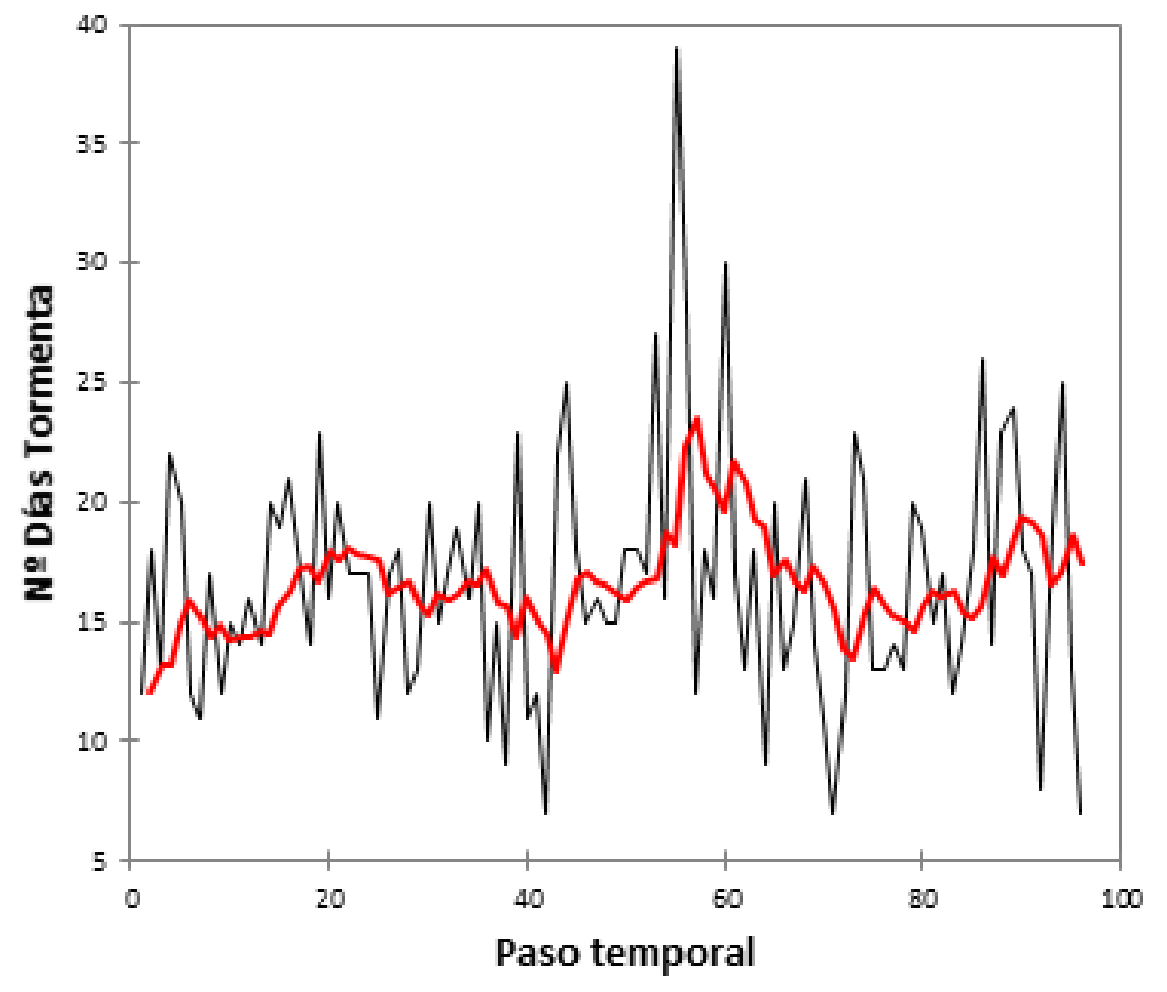

FUENTE: Elaboración propia.

La distribución de frecuencias es unimodal y el histograma tiene una forma acampanada nítida (FIGURA 3), pero la serie no puede considerarse normal, para un nivel de significación de 0,05 , según los tests de Lilliefors $(\mathrm{p}<0.006)$, Anderson-Darlin $(\mathrm{p}<0.018)$ y Shapiro-Wilk $(\mathrm{p}<0.001)$. Eso se debe a una notable curtosis (coeficiente de curtosis de Pearson de 2,4) y un sesgo positivo (coeficiente de sesgo de Pearson de 1,0).

En cambio, la distribución de frecuencias empírica, aun siendo discreta, admite un ajuste mediante la distribución GEV (Generalized extreme value), de parámetros $\mathrm{k}=0,028, \beta=4,216 \mathrm{y} \mu=14,391$ (FIGURA 4). 
Figura 3. Histograma de frecuencias relativas de la serie del número anual de días de tormenta del observatorio Fabra (Barcelona) (1917-2012)

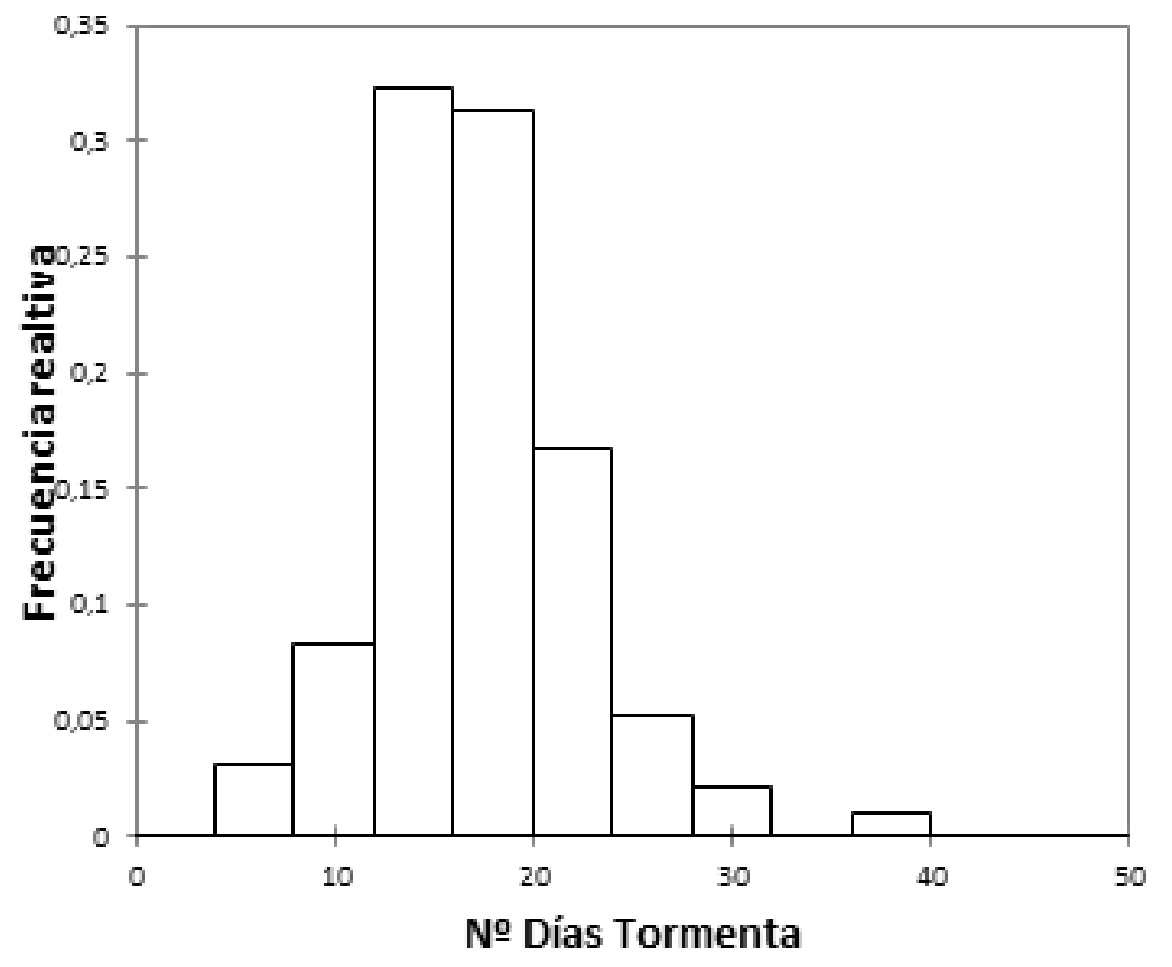

FUENTE: Elaboración propia.

Para garantizar un análisis estadístico temporal correcto hay que verificar previamente, como se dijo en el primer apartado, la homogeneidad de la serie. Para ello se han aplicado tres de los tests de homogeneidad más utilizados, los de Von Neumann, SNHT y Pettitt. Los tres, planteada la hipótesis nula de que la serie de 96 años es homogénea, y para un nivel de significación de 0,05 , admiten la misma $(\mathrm{p}=0.127 ; \mathrm{p}=0.626$ y $\mathrm{p}=0.843$, respectivamente). Por tanto, confirmada la homogeneidad de la serie, se está ahora en condiciones de estudiar las posibles tendencias en la misma.

Para determinar si el número anual de días de tormenta en el observatorio Fabra ha aumentado o disminuido significativamente, se ha aplicado el test de Mann-Kendall para dos colas y un nivel de significación de 0,05, con la hipótesis nula de que la serie es homogénea. Recuérdese que este test no requiere que la serie sea normal. El resultado es que no se puede rechazar la hipótesis nula ( $\mathrm{p}=0.792)$. En consecuencia, no ha habido cambio estadísticamente significativo en la frecuencia anual de las tormentas en el observa- 
torio barcelonés a lo largo de casi un siglo. Se ha repetido el análisis con la serie de 62 años que cubre la segunda mitad del siglo XX y los 12 años del siglo actual, de 1951 a 2012, comprobándose, igualmente, que es homogénea y no presenta tendencia. Parece, en conclusión, que el calentamiento reciente, bien constatado en el área de Barcelona, de $+0,2^{\circ} \mathrm{C} /$ década desde 1950 (BAIC, 2008), no ha tenido incidencia en la frecuencia anual de tormentas, que no ha variado en Barcelona.

Figura 4. Ajuste mediante la distribución GEV de la serie del número anual de días de tormenta del observatorio Fabra (Barcelona) (1917-2012)

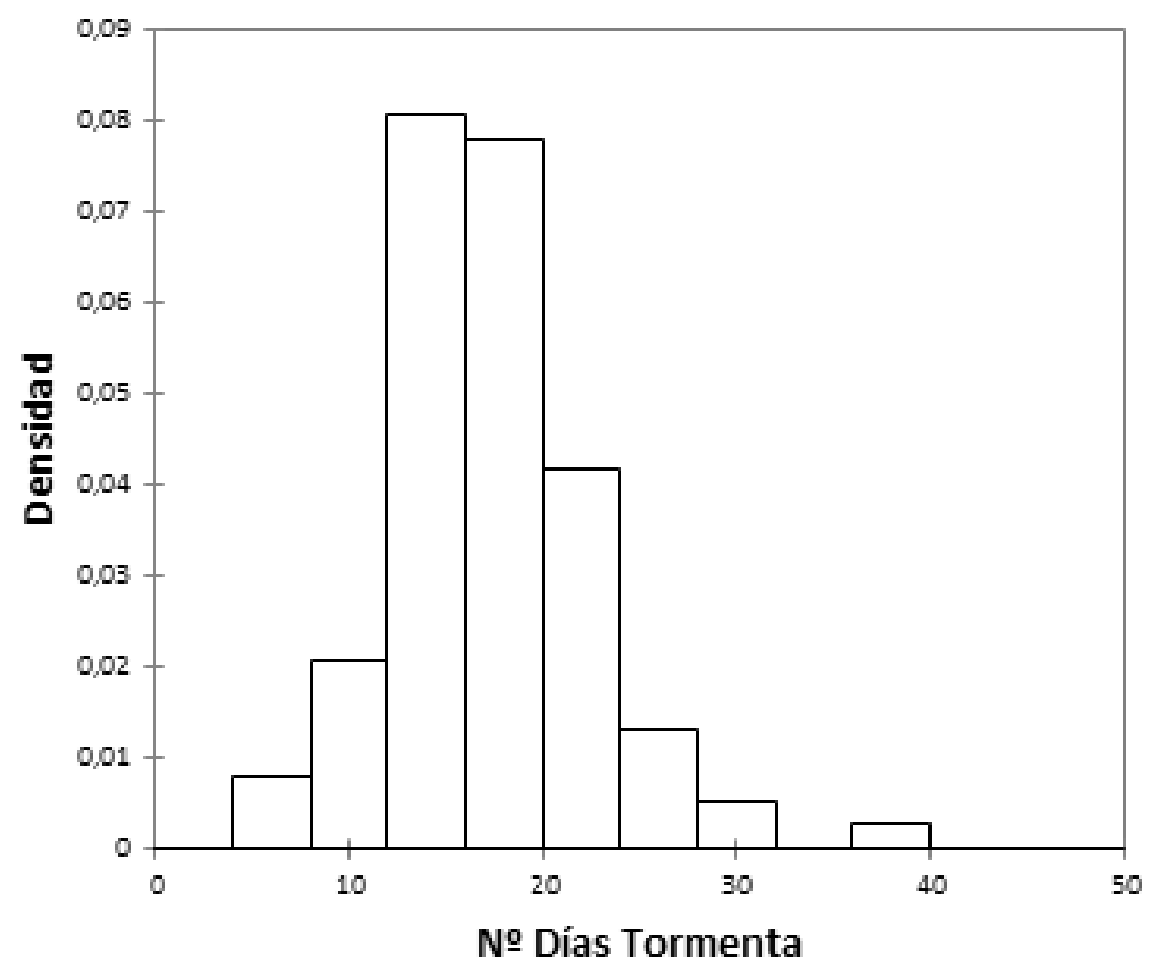

FUENTE: Elaboración propia.

\section{CONCLUSIONES}

La homogeneidad de una serie climática, que puede comprobarse mediante los tests homónimos, es un requisito previo e indispensable para poderla analizar cronológicamente, con el objeto de determinar sus posibles tendencias.

La definición de día de tormenta como aquel en que se oye el trueno lleva consigo en sí misma la imprecisión del límite de su audición en ambientes 
ruidosos o en horario intempestivo, lo que ha de dar lugar a series frecuentemente inhomogéneas.

Al comparar los promedios anuales de días de tormenta de los períodos 1931-1960 y 1971-2000 de 62 observatorios españoles de primer orden de la red de AEMET se aprecia un acusado y casi general incremento entre el primero y el segundo período, que, por la magnitud del mismo, no puede atribuirse a cambio o variación climática.

Una serie homogénea del número anual de días de tormenta de 96 años del observatorio Fabra (Barcelona), con una media de 16,7 días y una desviación tipo de 5,2 días, no ha mostrado tendencia alguna en el período 1917-2012, según el test de Mann-Kendal con un nivel de significación de 0,05.

\section{AGRADECIMIENTOS}

El trabajo se encuadra en el proyecto CGL2011-29263-C02-01 (Ministerio de Economía y Competitividad), en el marco del Grupo de Climatología 2009 SGR 443 (Generalitat de Catalunya) y en las líneas de interés del Comité Español del WCRP y de l'Institut de l'Aigua (Universidad de Barcelona). A Marc Prohom, por el suministro de la serie del observatorio Fabra.

\section{BIBLIOGRAFÍA}

AEMET: Valores climatológicos normales, 1971-2000. En línea: http://www. aemet.es/es/serviciosclimaticos/datosclimatologicos/valoresclimatologicos

Aguilar, E.; Auer, I.; Brunet, M.; Peterson, T.C. y WieringA, J. (2003): Guidelines on climate metadata and homogenization. Geneva, World Meteorological Organization, WMO-TD No. 1186.

AlEXANDERSSON, H. (1986): «A homogeneity test applied to precipitation data». International Journal of Climatology, vol. 6 (6), 661-675.

AleXANDERsson, H. y MoBerg, A. (1997): «Homogenization of Swedish temperature data. Part I: Homogeneity test for linear trends». International Journal of Climatology, vol. 17 (1), 25-34.

Auer, I.; BöHM, R.; Jurkovic, A.; ORlik, A.; Potzmann, R.; SchÖner, W.; UngersböCK, M.; BRUnetTI, M.; NANNI, T.; MAUgeRI, M.; BRIFFA, K.; JoNES, P.; EfTHYMiadis, D.; Mestre, O.; Moisselin, J-M.; Begert, M.; BRAZDil, R.; BOCHNiceK, O.; CEgnAR, T.; GAJIC-CAPKA, M.; ZANinOVIC, K.; MAJSTOROVIC, Ž.; SZALAI, S.; SZENTIMREY, T. y MERCALLI, L. (2005): «A new instrumental precipitation dataset for the greater alpine region for the period 1800-2002». International Journal of Climatology, vol. 25 (2), 139-166.

BAIC (2008): Butlletí Anual d'Indicadors Climàtics. Barcelona, Servei Meteorològic de Catalunya.

BUISHAND, T.A. (1982): «Some methods for testing the homogeneity of rainfall records». Journal of Hydrology, 58, 11-27. 
CostA, A.C. y SoAres, A. (2009): «Homogenization of Climate Data: Review and New Perspectives Using Geostatistics». Mathematical Geosciences, vol. 41 (3), 291-305.

CRADDOCK, J.M. (1979): «Methods of comparing annual rainfall records for climatic purposes». Weather, vol. 34 (9), 332-346.

DuCRÉ-RobitAille, J.F.; VinCENT, L.A. y Boulet, G. (2003): «Comparison of techniques for detection of discontinuities in temperature series». International Journal of Climatology, vol. 23(9), 1087-1101.

EAsterlinG, D.R. y PETERSON, T.C. (1995): «A new method for detecting and adjusting for undocumented discontinuities in climatological time series». International Journal of Climatology, vol. 15 (4), 369-377.

GonZÁlez MÁRqueZ, J.: Climatología de tormentas en España, publicado en www.meteored.com (2006) y en www.divulgameteo.com

HIRSCH, R.M. y SLACK, J.R. (1984): «A nonparametric trend test for seasonal data with serial dependence». Water Resources Research, vol. 20(6), 727-732.

INM (1982): Guía resumida del Clima en España. Madrid, Instituto Nacional de Meteorología.

KENDALL, M.G. (1975): Rank correlation methods. London, Charles Griffin.

KRUSKAL, W.H. y WALLIS, W.A. (1952): «Use of ranks in one-criterion variance analysis». Journal of the American Statistical Association, 47, 583-621.

MANN, H.B. (1945): «Non-parametric test against trend». Econometrika, 13, 245259.

MANN, H.B. y WhitneY, D.R. (1947): «On a test of whether one of two random variables is stochastically larger than the other». The Annals of Mathematical Statistics, 18, 50-60.

MARTÍN VIDE, J. (2003): El tiempo y el clima. Barcelona, Rubes.

MENNE, M.J. y WILLIAMS, C.N. (2005): «Detection of undocumented changepoints using multiple test statistics and composite reference series». Journal of Climate, vol. 18(20), 4271-4286.

Peterson, T.C.; EAsterling, D.R.; Karl, T.R.; Groisman, P.; NiChOlls, N.; Plummer, N.; TOROK, S.; Auer, I.; BOehM, R.; GulletT, D.; Vincent, L.; Heino, R.; TuOmenvirta, H.; Mestre, O.; Szentimrey, T.; Salinger, J.; Forland, E.J.; HANSSEN-BAuer, I.; AleXANDERSSON, H.; JONES, P.; PARKER, D. (1998): «Homogeneity adjustments of in situ atmospheric climate data: A review». International Journal of Climatology, vol. 18 (13), 1493-1517.

PETTIT, A.N. (1979): “A non-parametric approach to the change-point detection”. Journal of the Royal Statistical Society. Series C (Applied Statistics), 28(2), 126135.

ReEves, J.; Chen, J.; WANG, X.L.; Lund, R. y LU, Q. (2007): «A review and comparison of changepoint detection techniques for climate data». Journal of Applied Meteorology and Climatology, vol. 46 (6), 900-915.

SoLOW, A. (1987): «Testing for climatic change: an application of the two phase regression model». Journal of Applied Meteorology, vol. 26 (10), 1401-1405.

SZENTIMREY, T. (2003): «Multiple analysis of series for homogenization (MASH); Verification procedure for homogenized time series» en Fourth seminar for ho- 
mogenization and quality control in climatological databases. Budapest, Hungary, WMO-TD No. 1236, 193-201.

THOM, H.C.S. (1966): Technical Report No. 81, Geneva, WMO.

VINCENT, L. (1998): "A technique for the identification of inhomogeneities in Canadian temperature series». Journal of Climate, 11, 1094-1104.

Von NEumanN, J. (1941): «Distribution of the ratio of the mean square successive difference to the variance». Annals of Mathematical Statistics, vol. 12 (4), 367395.

WiJngaARD, J.; KLein TAnK, A.M.G. y Können, G.P. (2003): «Homogeneity of 20th century European daily temperature and precipitation series». International Journal of Climatology, vol. 23(6), 679-692.

WILCOXON, F. (1945): «Individual comparison by ranking methods». Biometrics, vol. 1 (6), 80-83. 
Anexo 1. NÚMERO ANUAL DE DÍAS DE TORMENTA EN EL OBSERVATORIO FABRA (BARCELONA), EN EL PERÍODO 1917-2012. FUENTE: OBSERVATORIO FABRA, REAL ACADEMIA DE CIENCIAS Y ARTES DE BARCELONA.

$\begin{array}{llll}\text { Año } & N^{o} \text { días } & \text { Año } & N^{o} \text { días } \\ 1917 & 12 & 1955 & 23 \\ 1918 & 18 & 1956 & 11 \\ 1919 & 13 & 1957 & 12 \\ 1920 & 22 & 1958 & 7 \\ 1921 & 20 & 1959 & 22 \\ 1922 & 12 & 1960 & 25 \\ 1923 & 11 & 1961 & 18 \\ 1924 & 17 & 1962 & 15 \\ 1925 & 12 & 1963 & 16 \\ 1926 & 15 & 1964 & 15 \\ 1927 & 14 & 1965 & 15 \\ 1928 & 16 & 1966 & 18 \\ 1929 & 14 & 1967 & 18 \\ 1930 & 20 & 1968 & 17 \\ 1931 & 19 & 1969 & 27 \\ 1932 & 21 & 1970 & 16 \\ 1933 & 18 & 1971 & 39 \\ 1934 & 14 & 1972 & 28 \\ 1935 & 23 & 1973 & 12 \\ 1936 & 16 & 1974 & 18 \\ 1937 & 20 & 1975 & 16 \\ 1938 & 17 & 1976 & 30 \\ 1939 & 17 & 1977 & 17 \\ 1940 & 17 & 1978 & 13 \\ 1941 & 11 & 1979 & 18 \\ 1942 & 17 & 1980 & 9 \\ 1943 & 18 & 1981 & 20 \\ 1944 & 12 & 1982 & 13 \\ 1945 & 13 & 1983 & 15 \\ 1946 & 20 & 1984 & 21 \\ 1947 & 15 & 1985 & 14 \\ 1948 & 17 & 1986 & 11 \\ 1949 & 19 & 1987 & 7 \\ 1950 & 16 & 1988 & 12 \\ 1951 & 20 & 1989 & 23 \\ 1952 & 10 & 1991 & 13 \\ 1953 & 15 & & 13 \\ 1954 & 9 & & \end{array}$




$\begin{array}{llll}\text { Año } & N^{o} \text { días } & \text { Año } & N^{o} \text { días } \\ 1993 & 14 & 2003 & 14 \\ 1994 & 13 & 2004 & 23 \\ 1995 & 20 & 2005 & 24 \\ 1996 & 19 & 2006 & 18 \\ 1997 & 15 & 2007 & 17 \\ 1998 & 17 & 2008 & 8 \\ 1999 & 12 & 2009 & 19 \\ 2000 & 14 & 2010 & 25 \\ 2001 & 18 & 2011 & 13 \\ 2002 & 26 & 2012 & 7\end{array}$

